\title{
Methicillin-resistant Staphylococcus aureus: a four-year experience in a spinal cord injury unit in Spain
}

\author{
M Olona-Cabases ${ }^{2}$, N Ticó-Falguera ${ }^{1}$, L Ramírez-Garcerán ${ }^{1}$, O Del Valle-Ortiz ${ }^{3}$, T Castelló-Verdú ${ }^{1}$ and \\ L García-Fernández ${ }^{1}$ \\ ${ }^{1}$ Spinal Cord Injury Unit, Traumatology and Rehabilitation University Hospital, Ciutat Sanitària Universitària Vall \\ d'Hebron, Psg. Vall d'Hebron 119-129, 08035 Barcelona, Spain; ${ }^{2}$ Preventive Medicine and Epidemiology Service \\ Ciutat Sanitària Universitària Vall d'Hebron; ${ }^{3}$ Microbiology Service, Ciutat Sanitària Universitària Vall d'Hebron
}

Methicillin-Resistant Staphylococcus aureus (MRSA) infection poses a problem for both acute and long-term-care facilities, Spinal Cord Injury units included. This paper describes the 4year evolution of MRSA outbreaks in a SCI unit in a university hospital where control measures were implemented from the first case detected. The protocol procedure was as follows: contact isolation, washing with antiseptic soap both those infected and those sharing the same room, contacts study and monitoring of MRSA patients up to the time when three consecutive negative cultures (sampled at time lapses of over $48 \mathrm{~h}$ ) were obtained, antiseptic soap for the health-care personnel to wash their hands, and cultures of the nares done on the personnel in the event of an outbreak.

Twenty-one (3.4\%) MRSA positive cases were detected out of 550 admissions registered during the study period (November 1990 through October 1994). The evolution occurred in three outbreaks and six isolated MRSA positive patients without secondary cases. $71.5 \%$ of the cases were nosocomial. Seven (33\%) were colonizated and $14(67 \%)$ infected. The 14 patients infected presented 15 infections: nine with urinary tract infections, three surgical wound infections, two tracheostomy wound infections, and one patient with a decubitus ulcer infection. Two of those with urinary tract infections presented with secondary sepsis. No carriers were detected amongst the personnel. Urinary tract colonizations responded to treatment with cotrimoxazol except in two cases in which combined treatment was required (cotrimoxazol plus rifampicin). The patients with a MRSA positive tracheal aspirate were negative after combined treatment. Wounds and cultures of the nares responded favorably to initial treatment. One of the patients with a urinary tract infection and sepsis died the infection being a contributing cause.

The prospective follow-up of the patients with MRSA positive cultures and the precocious implementation of isolation measures allow for the limitation of transmission, even although complete eradication is not possible.

Keywords: methicillin-resistant Staphylococcus aureus (MRSA); spinal cord injury; control measures

\section{Introduction}

In the last few decades Methicillin-Resistant Staphylococcus aureus (MRSA) has been the cause for an important number of outbreaks in hospitals, mostly tertiary hospitals, ${ }^{1}$ although other episodes have been described in long-term (LTC) facilities and spinal cord injury (SCI) units. ${ }^{2-5}$

The existing recommendations to control these outbreaks call for the enforcement of painstaking efforts and costly resources bearing in mind that, in

Correspondence: L García-Fernández certain cases, their efficacy becomes a controversial issue. Some authors have argued that it is not useful nor necessary to allocate any special effort towards control. We find, however, that in certain circumstances, such as the first case detected in a hospital, or in an outbreak setting, ${ }^{6}$ the infections cannot go untackled.

The purpose of this paper is to assess the control measures implemented in a SCI Unit within a medical university hospital, together with the follow-up and the treatment processes of such cases from the time of the first isolation of MRSA. 


\section{Materials and methods}

The population studied

Our SCI Unit is set within a 300-bed medical university hospital specializing in traumatology, rehabilitation therapy and neurosurgery (TRUH), it also includes an Intensive Care Unit (ICU) and is one of a three building complex called Ciutat Sanitària Universitaria Vall d'Hebron also having a medical-surgical general hospital and a maternity-pediatric hospital.

The 30-bed SCI Unit caters for acute and long-term cases and is divided into 6, 3 and 2-bed rooms. Trauma is the commonest cause for a patient's admission. Acute patients are admitted directly into the unit, or are referred via our own ICU, and some from external sources.

Duration of the study

From November 1990 through to October 1994.

\section{Control regimen}

As from 1971 the Bacteriology Service in the Ciudat Sanitària Universitaria Vall d'Hebron manages a follow-up program of MRSA isolation in each facility. On identification of a case, isolation measures are immediately implemented and the Preventive Medicine Service carries out the epidemiological control which is outlined, depending on the characteristics of the unit where the case is identified, with a protocol based on the recommendations issued by the Hospital Infection Society and the British Society for Antimicrobial Chemotherapy.

The following are the measures implemented by this SCI Unit:

1. Isolation measures: On identifying a case, hospital discharge is carefully considered with the patient undergoing home treatment. Should discharge not be possible the patient has contact isolation and is placed in a private room, except for those cases in whom only the cultures of the nares are MRSA positive. In the event of clustering various patients can share a room.

Hygiene of the MRSA positive cases and of those sharing the same room is made by means of chlorhexidine soap bathing for one week. Chlorhexidine soap is also used by attending personnel to wash their hands. The room is cleaned with an aldehyde solution.

Such isolation measures are used throughout until the patient's cultures are negative for MRSA.

2. Epidemiological control On admission, cultures are taken of patient's urine, wounds, ulcers, and tracheostomy wounds (when present).

When a case is identified, cultures are also taken from other patients who have been in contact with the patient concerned. Samples are obtained from the urine, nares, wounds, ulcers, and tracheostomy wounds. If the patient's temperature readings are above $38^{\circ} \mathrm{C}$ consecutive hemocultures are performed.
When two or more cases appear simultaneously, the cultures mentioned above are taken from all of the patients registered in the unit, and cultures of the nares are performed of health-care personnel.

On completion of the course of treatment after obtaining favourable antibiogram results, patients previously positive for MRSA are considered to be fully recovered after obtaining three consecutive negative cultures of each site, taken at $48 \mathrm{~h}$ intervals.

\section{Case definition}

A patient with at least one MRSA-positive culture is defined as a case.

Infections are diagnosed following the criteria established by the CDC (Center for Disease Control) ${ }^{8}$ amended for urinary tract infection (UTI) given the characteristics of these patients. A UTI is diagnosed when a MRSA-positive urine culture is found in conjunction with fever which does not originate in any other infection foci.

Patients who do not meet infection criteria are considered to be colonized.

The identification of a culture as newly positive after three consecutive negative results is considered as a relapse.

An infection is considered nosocomial if MRSApositive cultures are obtained within $48 \mathrm{~h}$ of admission in the SCI Unit, unless there is sound knowledge that the patient was a carrier prior to hospital admission.

The identification of two or more nosocomial cases in the unit is considered an outbreak. The follow-up period was set at 3 months.

\section{Bacteriological study}

In order to identify Staphylococcus aureus the usual methods are used. The antibiogram is performed by disk-elution (autobac), Vitak or microdilution (ALADIN) methods. Oxacillin resistance is determined by

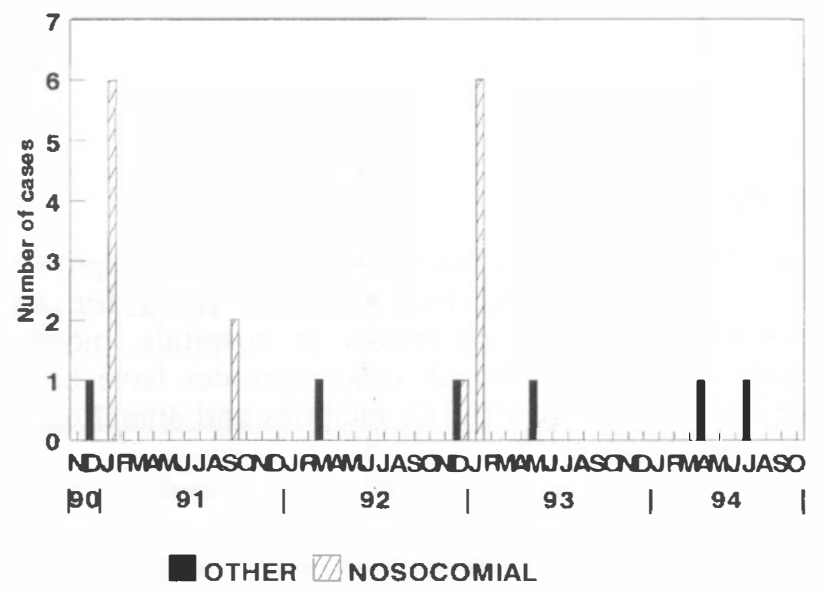

Figure 1 MRSA evolution in SCI unit Novermber 1990October 1994 
Mueller Hinton agar diffusion with added $\mathrm{NaCl}$ at $35^{\circ} \mathrm{C}$ for $24 \mathrm{~h}$ with $25 \mathrm{mg}$ oxacillin pills checked-up with microdilution following the NCCLS (National Committee of Clinical Laboratory Standards) method. ${ }^{9}$ Phagogroups were determined at the CNMVIS (Centro Nacional de Microbiología, Virología e Inmunología Sanitarias) Majadahonda, Madrid. This centre uses an international group of phages standardized at the routine test dilution or at $100 \times$ RTD. If they do not type, the reverse phagotypy method is used after heat treatment. $^{10}$ We also used a group of nine phages, numbered 30-38, obtained by the centre from the Spanish MRSA samples, and considered three patterns on the basis of the phages that produce lysis.

\section{Results}

Outbreak description and evolution

Figure 1 shows the evolution of three outbreaks which coincided with the admission to the Unit of an MRSApositive patient. Six isolated MRSA positive patients without secondary cases were described as well, the last two in 1994.

The first two outbreaks originated in the Centre's ICU, and in the second outbreak the neurosurgery ward was also involved. The third outbreak was isolated in the SCI Unit and was due to the admission of one MRSA-positive patient referred to us from an external ICU who was not identified as an MRSA carrier on admission and who was not isolated until the results of the culture performed in the unit were available.

The antimicrobial resistance detected is shown in Table 1. Oxacillin minimal inhibition concentration (MIC) was equal to or greater than $128 \mathrm{mg} / \mathrm{l}$. The antibiotype was the same, in the first two outbreaks: resistance to aminoglycosides, except for amikacine, macrolyds, glucosamines, fluoroquinolones and susceptibility to chloramphenicol, fosfomycine, vancomycin, cotrimaxozol, and fusidic acid, with slight differences according the technique used. The strains

Table 1 Antimicrobial resistances of the strains isolated

\begin{tabular}{lcccr}
\hline $\begin{array}{l}\text { Antibiotics } \\
R\end{array}$ & Tested & $S$ & $M$ & \\
\hline Gentamicine & 53 & 7 & 0 & 46 \\
Tobramicine & 53 & 0 & 0 & 53 \\
Amikacin & 53 & 35 & 5 & 13 \\
Chloramphenicol & 35 & 34 & 1 & 0 \\
Erythromycin & 52 & 0 & 0 & 52 \\
Lincosamines & 52 & 0 & 0 & 52 \\
Fosfomicine & 36 & 36 & 0 & 0 \\
Vancomycin & 53 & 53 & 0 & 0 \\
Cotrimoxazol & 53 & 50 & 1 & 2 \\
Norfloxacine & 53 & 0 & 4 & 49 \\
Ciprofloxacine & 52 & 0 & 4 & 48 \\
Fusidic acid & 28 & 26 & 0 & 2 \\
\hline
\end{tabular}

$\mathrm{S}=$ susceptible; $\mathrm{M}=$ medium; $\mathrm{R}=$ resistant isolated in the third outbreak showed a somewhat different antibiotype. The oxacillin MIC ranged from 32 to $64 \mathrm{mg} / \mathrm{l}$, and strains were susceptible to gentamicine but resistant to tobramicine and amikacine.

Most of the strains isolated were MRSA phage type III or non-typable by reverse phagotypy. The nontypable strains all presented the same pattern of lysis. The strains isolated in the third outbreak presented phage type 95 . No health-care personnel were MRSApositive.

Case description

The total number of patients with MRSA-positive cultures from November 1990 through October 1994 was 21 out of 550 admissions (incidence: $3.4 \%$ in 4 years).

All cases but one were men; mean age was 43 (Standard Deviation 19; extreme ages: 18 and 81). In Table 2 we describe the general characteristics of these patients. Of the 21 patients, MRSA was isolated in 12 in the urine $(57.1 \%), 11$ in the nose $(52.3 \%)$, three in surgical wounds $(14.3 \%)$, two in tracheostomy aspirate $(9.5 \%)$, one in a decubitus ulcer $(4.7 \%)$ and two Hemoculture $(9.5 \%)$. The urine samples were taken in 15 patients with indwelling urinary catheters with a closed sterile drainage system, and six with intermittent catheterization. All positive MRSA isolations were in patients with indwelling catheters.

Out of the total number of 21 cases, $15(71.5 \%)$ were considered nosocomial. Seven $(33 \%)$ were considered colonized and $14(67 \%)$ infected.

Table 2 Case description

\begin{tabular}{lcr}
\hline & No. & $(\%)$ \\
\hline Admission cause & & \\
Road accident & 11 & $(52.4)$ \\
Work accident & 3 & $(14.3)$ \\
Sport accident & 3 & $(14.3)$ \\
Fortuitous fall & 2 & $(9.5)$ \\
Decubitus ulcer & 2 & $(9.5)$ \\
Lesion site & & \\
Cervical & 9 & $(43)$ \\
Thoracic & 8 & $(38)$ \\
Lumbar & 2 & $(9.5)$ \\
Sacrum & 2 & $(9.5)$ \\
Lesion phase & & \\
Acute & 9 & $(43)$ \\
Subacute & 8 & $(38)$ \\
Chronic & 4 & $(19)$ \\
Patients' origin & & \\
Hospital ICU & 3 & $(14.3)$ \\
External ICU & 5 & $(23.8)$ \\
Direct admission & & \\
$\quad$ to SCI unit & 13 & $(61.9)$ \\
\hline
\end{tabular}

No. $=$ total number of patients 
In the 7 colonized patients, MRSA was isolated in cultures of nares in four cases and in urine cultures in three.

The $14(67 \%)$ infected patients presented 15 infection procedures: nine presented UTI (two with secondary septicemia), three presented postsurgical wound infection, one presented decubitus ulcer infection and two tracheostomy wound infection.

Of the cases with UTI without secondary sepsis, five $(62 \%)$ presented with an association with gram negative bacilli (GNB), which makes it difficult to determine whether the infection was caused by this bacteria or by MRSA since subjects responded to treatment with combined antibiotics. In the two patients with UTI associated with secondary sepsis, the urine cultures were only positive for MRSA.

The treatment of infections was established on the basis of the antibiogram. Urinary tract infections were treated with amikacine, except for the cases presenting associated sepsis in which vancomycin was used. Colonizations were treated with cotrimoxazol which, in the event of failure, was combined with rifampicin or replaced by fosfomicine. The cases with MRSApositive cultures of the nares were treated with topical mupirocin for 5 days.

Evolution

- The patients with nasal colonization were negative after topical treatment with mupirocin.

- The cases with postsurgical wound infection and decubitus ulcer showed a successful outcome after receiving appropriate treatment.

- Patients with tracheostomy infection were negative after combined rifampicin and cotrimoxazol treatment.

- Of the 12 patients with positive urine cultures, two $(16 \%)$ relapsed and one $(8.3 \%)$ died, the infection being one of the contributing causes. Follow-up was discontinued in one case as the patient was transferred to another institution.

\section{Discussion}

Most of the patients in whom MRSA was isolated in the urine $(75 \%)$ developed infection, though it was difficult to identify the causative agent in those patients who presented an association with GNB. In these cases antibiotic treatment for both infections was chosen.

Currently treatment of urine colonizations is not considered appropriate in patients with Spinal Cord Injury. ${ }^{11}$ In cases of MRSA colonization, treatment is justified in order to prevent the recurrence of infection, or to stop transmission. ${ }^{12}$ We decided to treat patients in order to stop transmission and to avoid prolonged isolations which hinder the rehabilitation process, have a negative psychological effect on these patients and extend hospitalization excessively. Nonetheless, as we did not have a control group of colonized patients not undergoing antibiotic treatment, it was impossible to assess the efficiency of the treatment.
The effects of MRSA in the urine did not differ from that of other habitual germs in patients with a neuropathic bladder, and sometimes resulted in severe infections. Thus, out of the 12 patients with MRSApositive urine cultures, nine developed infection, two of them with secondary septicemia.

As is described in the literature ${ }^{12}$ the combination of rifampicine with cotrimoxazol was effective in making cultures negative when cotrimoxazol alone had not been successful. In any case, the follow-up period was too short for us to draw any conclusions on the longterm evolution.

Although patients with positive cultures only of the nares were not isolated in private rooms, no increase in the number of cases was observed. This finding supports the hypothesis that MRSA is transmitted basically via the hands of the attending personnel. ${ }^{1}$

In every case, mupirocin was effective in making all of the cultures of the nares negative. MRSA epidemics have been documented in Spanish hospitals since 1988 and at times have affected a large number of patients including carriers amongst the health-care personnel. ${ }^{13}$ In our unit, outbreaks were self-limited, and no colonization or infection was identified in the healthcare personnel. Early detection and immediate control measures are probably the reason for this, even although the isolations identified in our unit fully matched the Spanish epidemic strain. However, given the situation in our country, it is difficult to eradicate the organism from a hospital although its spread can be controlled.

In this period a change in the resistance patterns was seen in isolations. In the first two outbreaks the strains were susceptible to amikacine and treatment with it proved to be successful, but in the third the strains were amikacine resistant. No cases of resistance to vancomycin, rifampicin, fosfomicine or chloramphenicol were found.

\section{Conclusions}

MRSA behaviour in the urine shows no differences from that of other germs. The number of cases does not increase if the patients with MRSA positive cultures of the nares and other cultures negative are not isolated in private rooms.

Antibiotics association is useful when confronted with non-negativization. Health-care personnel were never infected. The prospective follow-up of the patients with MRSA positive urine cultures and the precocious implementation of isolation measures allow for the limitation of transmission, even although complete eradication is not possible.

\section{Acknowledgements}

We thank Josep Graells for manuscript preparation and linguistic assessment. All of the health-care workers in the SCI unit at TRUH. 


\section{References}

1 Mulligan ME et al. Methicillin-resistant Staphylococcus aureus: a consensus review of the microbiology, pathogenesis, and epidemiology with implications for prevention management. Am J Med 1993; 94: 313-328.

2 Bradley $\mathrm{S}$ et al. Methicillin-resistant Staphylococcus aureus: colonization and infection in a long-term care facility. Ann Intern Med 1991; 115: 417-422.

3 Kauffman CA et al. Attempts to eradicate Methicillin-resistant Staphylococcus aureus from a long term care facility with the use of mupirocin ointment. Am J Med 1993; 94: 371-378.

4 Darouiche $\mathrm{R}$ et al. Eradication of colonization by MethicillinResistant Staphylococcus aureus by using oral Minocyclinerifampin and topical mupirocin. Antimicrob Agents Chemother 1991; 35: $1612-1615$.

5 Maeder K, Ginunas VJ, Montgomerie JZ, Canawati HN. Methicillin-resistant Staphylococcus aureus (MRSA) colonization in patients with spinal cord injury. Paraplegia 1993; 31: $639-644$.

6 Boyce JM. Should we vigorously try to contain and control Methicillin-resistant Staphylococcus aureus? Infect Control Hosp Epidemiol 1991; 12: 46-54.
7 Working Party Report. Revised guidelines for the control of epidemic methicillin-resistant Staphylococcus aureus. J Hosp Infect 1990; 16: 351 - 377 .

8 Garner JS et al. CDC definitions for nosocomial infections, 1988. Am J Infect Control 1988; 16: 128 - 140.

9 NCCLS: Methods for dilution antimicrobial susceptibility test for bacteria that grow aerobically. Third edition; Approved standard NCCLS document M7-A3. NCCLS, Vilanova 1993.

10 Saxe WJ, Notley. Experience with typing of coagulase negative Staphylococcus and Micrococcus. Zbl Backt n Hyg 1978; 241: $46-59$.

11 Stamm WE. Nosocomial urinary tract infections. In: Bennett JV, Brachman PS. Hospital infections. Third Edition. Little, Brown and Company. Boston, 1992.

12 Chambers HF. Treatment of infection and colonization caused by Methicillin-resistant Staphylococcus aureus. Infect Control Hosp Epidemiol 1991; 12: 29-35.

13 Trilla A, Wenzel RP. Infecciones nosocomiales por Staphylococcus aureus resistentes a la meticilina. Un reto para el control de infecciones. Enf Infec y Microbiol Clin 1991; 9: 193-195. 